\title{
Purkinje cells and autism spectrum disorder. Is there an aetiological connection?
}

\author{
Kamil D. Lucci \\ Department of Child Psychiatry, Warsaw Medical University, Poland \\ School of Psychology and Neuroscience, University of St Andrews, United Kingdom \\ Neuropsychiatria i Neuropsychologia 2018; 13, 2: 57-64
}

\author{
Address for correspondence: \\ Kamil D. Lucci \\ Department of Child Psychiatry \\ Warsaw Medical University \\ Żwirki i Wigury 61 \\ 02-091 Warsaw, Poland \\ e-mail: dante lucci@hotmail.com
}

\begin{abstract}
Autism spectrum disorder (ASD) is a neurodegenerative disorder affecting the quality of life of a significant number of children and adults around the world. Despite its growing epidemiology, the causes of autism are still not known. Research has linked the disorder with brain pathology, with a possible prenatal onset. It has been reported that the brain area involved in autism is the cerebellum, and more specifically, a disruption within Purkinje cells. For this reason, Purkinje cells are among the most studied brain cells in relation to ASD. Despite the amount of research on the topic and different techniques applied, the aetiology of ASD is still unclear. One of the possible causes of the relationship between ASD and Purkinje cell abnormality might be their vulnerability. Studies suggest that Purkinje cell loss might occur early in life (in the prenatal or neonatal period) as a result of contact with toxins or brain damage. The aim of this review is to describe the most important findings in the field and discuss the aetiological connection between Purkinje cell loss and ASD. First, this review discusses the neurological basis of autism. Then, the evidence of the link between Purkinje cell damage and ASD, presented in such studies, is described. The main part of the article analyses major studies within the field. They have been grouped based on the research techniques applied: mutant mice models, histopathological examinations, and in vivo neuroimaging. At the end, the limitations of the study and future research possibilities are discussed.
\end{abstract}

Key words: Purkinje cells, autism, cerebellum, neurodegeneration.

\section{Introduction}

Autism spectrum disorder (ASD) is a pervasive developmental disorder characterised by social, linguistic, and sensorimotor deficits (McPartland et al. 2012). Because the number of children diagnosed with ASD has significantly grown in recent years, researchers seek the cause of ASD. For instance, in 1984 in the USA, only four in 10,000 children were diagnosed with autism (Ritvo et al. 1986), but the prevalence rose to six per 10,000 in 1994 , and 31 per 10,000 in 2003 (Shattuck 2006). There is consistent research trying to find a neurological basis for autism. ASD has been linked to the impairment of several brain areas such as hypoactivation of the inferior frontal gyrus (Dapretto et al. 2006), cortical thinning (Hardan et al. 2006; Wallace et al. 2010), enlarged amygdala (Munson et al. 2006), and transverse relaxation of grey matter (Petropoulos et al. 2006). Previous studies on autism have also found a connection between cerebellar dysfunction in people with ASD and a loss of Purkinje cell volume (Fatemi et al. 2002). Over the last two decades, a number of studies have proven this relationship in brains of many autistic patients (Ritvo et al. 1986; Bailey et al. 1998; Fatemi et al. 2002).

According to Galen, the cerebellum was first mentioned by Aristotle and Herophilus. However, Galen's research was the earliest account on the cerebellum, and he was the first to link the cerebellum with motor functions (Finger 2001). In the $16^{\text {th }}$ Century, Leonardo da Vinci researched brain structure and named one part of the brain the "cerebellum" (Gross 1997). The name given by da Vinci to the cerebellum evoked its small size. In fact, despite the cerebellum comprising only $10 \%$ of brain volume, it contains around half of the brain's neurons (Li et al. 2013). The cerebellum is composed of 
only six main types of cells (granule, purkinje, golgi, unipolar brush, basket, and stellate). Its organisation is very regular, processing similar signals in the same way (Voogd and Glickstein 1998). However, the exact role of the cerebellum is still being researched. Initially, it was believed that the role of the cerebellum was primarily balance and motor control, while the cerebral cortex was responsible for cognitive functions (Schmahmann and Sherman 1998). However, evidence suggests that the cause of the severity of malfunctions related to the cerebellum might lie with it receiving input from areas crucial for the body's functioning: the vestibular nerve (responsible for sensory input), the spinal cord (affecting muscle tone and crude movements), the pontine nuclei (regulating the cerebral cortical output), and the cerebral cortex (learning and regulating behaviours; Voogd and Glickstein 1998).

Despite the cerebellum being known at least since Late Antiquity, the discovery of Purkinje cells was only made in the mid-19 $9^{\text {th }}$ Century by Johannes Purkinje. This finding was crucial for today's understanding of the cerebellum. Purkinje cells were first noticed for their large size, and later research reported the crucial role they play in the functioning of the brain. Purkinje cells are situated in the middle layer of the cerebellar cortex, between the outer molecular and the inner granular layer. They send inhibitory projections to the deep cerebellar nuclei, and some Purkinje cells send axons directly to the vestibular nuclei located in the brainstem (Stoodley 2012). Further studies on those neurons have associated their role with delivering information from the cerebellum to the cerebral cortex (Schmahmann and Sherman 1998). They are said to both receive information from the outer layer of the cerebellum and send signals to the deep cerebellar nuclei in the centre of the cerebellum. Research on the role of the cerebellum and its Purkinje cells has helped increase the understanding of disorders such as Alzheimer's (Joachim et al. 1989) and autism (Fatemi et al. 2002). Because their malfunctioning affects functions known to play a role in ASD, researchers claim that Purkinje cells are closely linked to the aetiology of autism (Bailey et al. 1998). Despite the fact that this topic has been studied for over two decades, researchers met for the first time at the 2015 Society of Neuroscience conference to present and discuss the involvement of Purkinje cells in ASD (Role 2015).

Overall, a large number of studies have found loss of these cells in a large percentage of autism cases, especially those without a clear genetic aetiology (Ritvo et al. 1986; Fatemi et al. 2002). Because the aetiology of ASD is still not known, researchers search for an explanation that would enhance the treatment and prevention of ASD. Over the years, various techniques have been implemented in research on Purkinje cells and autism - studies using modified animals, post-mortem studies, and in vivo studies implementing the latest scanning methods. It is crucial to analyse the results of each of those perspectives.

\section{Cerebellum impairment and its link to autism}

Despite the fact that some studies are reporting an increase of cerebellar volume in autistic patients (Traut et al. 2018), most studies conclude that volume loss in the cerebellum is positively correlated with autism (Ritvo et al. 1986; Bailey et al. 1998; Fatemi et al. 2002). For instance, Courchesne et al. (1993) found that the posterior lobe (lobules VI and VII) and the vermis (lobules VIII-X) in the cerebellum were significantly smaller in autistic subjects.

However, Fatemi et al. (2002) reported in their study no changes in Purkinje cell density in autistic patients and only a decreased size of the Purkinje cell somata. In other studies, it was found that Purkinje cell numbers were significantly decreased (Ritvo et al. 1986; Bailey et al. 1998). Because autism exhibits a very broad range of severity, it is possible that Purkinje cell loss is only evident in the most severe cases.

\section{Neurological basis of autism}

An important number of post-mortem studies have reported cerebellar pathology in autistic brains (Ritvo et al. 1986; Bailey et al. 1998). Imaging studies have also reported increased cerebellar Purkinje cell volume loss in ASD patients (Ritvo et al. 1986; Fatemi et al. 2002).

In the past the only behaviours linked to the cerebellum were motor coordination and motor learning (Schmahmann and Sherman 1998). Thanks to the development of research on Purkinje cells, it is now believed that the cerebellum is not only a regulator of movement, but also the brain region responsible for cognitive functions such as language, visual and spatial understanding, auditory processing, verbal memory, fine motor regulation, and executive function (Hampson and Blatt 2015; Starowicz-Filip et al. 2013). Schmahmann and Sherman (1998) note that pathologies in the 
cerebellar brain area have been linked in research with patients' difficulties in abstract reasoning, working memory, and spatial cognition, as well as language deficits and disrupted verbal fluency. Some individuals with abnormal functioning of the cerebellum have also been reported to have behavioural issues similar to those identified in ASD (Schmahmann and Sherman 1998; Hampson and Blatt 2015). A healthy and properly functioning cerebellum is involved in behaviour modification (Schmahmann and Sherman 1998). In addition, pathologies in the cerebellum might have serious consequences. For instance, individuals with abnormalities in this brain area might exhibit cerebellar cognitive affective syndrome, a condition characterised by impaired executive functions, disrupted spatial cognition, blunted affect, inappropriate behaviour, and language deficits (Schmahmann and Sherman 1998). Furthermore, studies on animal models of ASD have been reported to show cerebellar abnormalities (Tsai et al. 2012; Reith et al. 2013; Cupolillo et al. 2016). Mutant mice (with an induced pathology in the cerebellar area) exhibited impaired motor deficits, and mental and behavioural abnormalities (Tsai et al. 2012; Reith et al. 2013). According to the fifth edition of the Diagnostic and Statistical Manual of Mental Disorders (APA 2014), autistic spectrum disorders present all of these characteristics (McPartland et al. 2012).

\section{Purkinje cell impairment in the cerebellum}

As mentioned above, the loss of Purkinje cells negatively influences the functioning of the frontal cortex. A study by Beversdorf $e t a l$. (2005) linked an early manifested ASD symptoms in children who born prematurely with a reported cerebellar injury. The role of Purkinje cells in the brain is closely related to the effect of their loss on human (and animal) behaviour (Sudarov 2013). Purkinje cells are reported to receive excitatory input from cerebellar granule cells, and in turn, using gamma-aminobutyric acid (GABA) as a neurotransmitter, provide inhibition to the deep cerebellar nuclei (Sudarov 2013). Then, the four deep nuclei send projections to brain areas vital for our functioning, such as the thalamus, and through it to the cerebral cortex (Sudarov 2013). This might influence the functioning of several mechanisms within the brain. For instance, Purkinje cell disruption might affect cerebellar modulation of dopamine release in the prefrontal cortex (Sudarov 2013). Then, it is possible that Purkinje cell pathology leads to an imbalance of the excitation and in- hibition processes in the cortex (Sudarov 2013). It has been hypothesised that this may be one of the mechanisms causing ASD because the abnormally functioning cortex is responsible for memory, perception, cognition, and motor control (Rubenstein and Merzenich 2003; Sudarov 2013; Yip et al. 2007).

However, it has also been hypothesised that the cause of Purkinje cell loss is their vulnerability. The effect of neonatal exposure to heavy metals and toxins on diminishing Purkinje cell numbers is widely discussed in the literature (Sowell et al. 1996; Kern and Jones 2006). However, Purkinje cells can also be affected by ineffective oxygen and blood supply (Au et al. 2015; Kaur et al. 2014), seizures (Krook-Magnuson et al. 2014), viral infections (Ludlow et al. 2016), or vitamin deficiencies (Ulatowski et al. 2014). One of the cells that might also be affected by the above-mentioned factors are the Bergmann glia cells that grow their bodies around Purkinje cells, and their functioning is strictly related to Purkinje cells (Yamada and Watanabe 2002; Chrobak and Soltys 2017). Thus, it is possible that neurodegeneration seen in ASD patients might have a more complex developmental deficit.

\section{Autopsy studies on brains of autistic individuals}

Decreased Purkinje cell density and/or number of Purkinje cells is a relatively consistent observation across post-mortem studies. Despite their importance, studies on the brains of deceased individuals tend to have very small sample sizes. For instance, Ritvo et al.'s (1986) paper presents one of the earliest studies seeking to determine the number of Purkinje cells in autistic brains. They examined the brains of only four 10-22-year-old autistic males and compared them with three 3-23-year-old male and one five-year-old female control/s. Despite their successful link between autism and decreased numbers of Purkinje cells, their findings would be more reliable if a larger sample size were used. Unfortunately, despite the fact that over 30 years have passed since Ritvo et al.'s (1986) study, no research has used a significant number of brains in their studies. Thus, the generalisability of their findings might be questioned.

Although Kemper and Bauman's (1993) article is one of the earliest studies on Purkinje cells in autistic brains, it is one of the most comprehensive studies using the autopsy technique. In the study, examination was performed 
of six brains of autistic patients in their 20s and 30s. All six cases were compared with controls of identical age and same sex proportions. The findings revealed pathologies in both the cerebellum and forebrain. However, Kemper and Bauman (1993) focused their research only on the cerebellar area. Similarly to other studies discussed in this article, the researchers found a significant decrease of Purkinje cell density in all tested brains. However, the density loss varied from $50 \%$ to $95 \%$. Interestingly, despite severe abnormalities in Purkinje cells, the cerebellar cortex was developed in all tested brains.

Kemper and Bauman (1993) hypothesised that in individuals who displayed typical infantile autism the cerebellar pathology might have its origins in the foetal stage. As predicted, the tested individuals seemed to have acquired the cerebellar lesions before being born. According to the authors, the cerebellar abnormalities appeared before the foetus entered its $30^{\text {th }}$ week of pregnancy. This finding suggests that ASD diagnosis might be made straight after birth. Because the changes in Purkinje cell structure was present in all studied cases, Kemper and Bauman (1993) concluded that ASD brains might be affected by cerebellar hypoplasia, a disorder of cerebellar maldevelopment.

Another early study reported similar findings to those obtained by Kemper and Bauman (1993). Bailey et al.'s (1998) study used post-mortem brain tissue obtained from six individuals with autism. It was discovered that not only Purkinje cell numbers were significantly reduced in all studied cases, but also that they presented more or less severe structural damage. The reduced number of Purkinje cells appeared all over the cerebellum area, their position was not regular, and the cell density was much lower than in healthy controls. However, Bailey et al. (1998) reported no empty baskets in the Purkinje cell tissue. Unfortunately, Bailey et al. (1998) did not report the patients' symptoms before death occurred. However, such changes in the structure have been connected to ASD symptoms (Bailey et al. 1998; Takashima 1982).

Similar findings were reported by Yip et al. (2007). Their research showed a significant reduction (approximately 40\%) of glutamate decarboxylase, an enzyme involved in the synthetisation of GABA, in all ASD individuals. As described earlier, such a finding links directly to the functioning of Purkinje cells that, without a properly functioning GABA, might disrupt the functioning of both motor and cognitive functions. Despite the importance of Yip et al.'s (2007) findings, similarly to the two earlier cited post-mortem studies, the research involved a very small sample of frozen brains (eight autistic and eight control patients).

Despite using a very small sample size of six autistic and four control cerebella, Whitney et al.'s (2009) study is very important in this research area because it was the first to use stereological cell counting data on the cerebella of ASD patients. Whitney et al.'s (2009) research confirmed the hypothesis made by Kemper and Bauman (1993). It seems that neuronal pathologies are not caused by postnatal injuries, but all abnormalities begin in the gestational period. Thus, Whitney et al. (2009) demonstrated that the cerebella of people diagnosed later in life with ASD already suffer neuronal loss. According to Whitney et al. (2009), Purkinje cell loss follows a similar pattern; however, the timing of such a process has to be further investigated. If such a hypothesis is be confirmed, investigating the processes underlying Purkinje cell loss (and other neurons) might be an interesting field with a lot of potential. Unfortunately, one major weakness of all of the discussed autopsy studies was their very small sample sizes. Thus, further research employing larger samples might show even more interesting results. It is also important to note that reduced numbers of Purkinje cells have been found in the cerebella of both children and adults. Thus, in the studies of both Kemper and Bauman (1993) and Bailey et al. (1998) the absence of glial hyperplasia has been reported. This finding would suggest that the cerebellar abnormalities have occurred early in development.

\section{Purkinje cells in autistic mutant mice}

The role of the cerebellum in autism remains a widely-researched topic, mainly because the link between abnormalities in social interactions and repetitive behaviours is still being investigated. Progress made in genetics in the last decade has provided the necessary tools to manipulate genes in animals and thus study complex neurological disorders. Using such techniques, Tsai et al. (2012) established a link between abnormal cerebellar Purkinje cell functioning and behavioural deficits that are relevant to ASD.

Recent animal studies have linked the Purkinje cell loss in the cerebellum to ASD, showing that not only humans are affected by their malfunctioning. However, the link between cerebellar dysfunction and ASD remains unclear; an important discovery was linking Purkinje cell loss to brain injuries. This opened the possibility 
of investigating the pathology on animal models. Tsai et al. (2012) in their research used a combination of several methods (mouse mutations, electrophysiology, behavioural tests, and pharmacological manipulations) to investigate the role of tuberous sclerosis (TSC) in Purkinje cell dysfunction in the cerebellum. Tuberous sclerosis complex is a genetic disorder with high comorbidity with the ASD. The link between TSC and autism is caused by mutations in either TSC1 or TSC2, whose protein regulates the mechanistic target of rapamycin (mTOR) signalling (inhibition of which leads to the development of TSC). TSC causes brain tissue malformations leading to a number of neurological symptoms such as intellectual disability, behavioural problems, developmental delays, and epilepsy. Studies have shown that more than half of TSC patients develop ASD. Despite the knowledge of the TSC gene, the aetiological connection between these two disorders is still not known.

Tsai et al. (2012) demonstrated that defects in Purkinje cell structure are positively correlated with autism. They discovered that mice with a TSC1 loss in their Purkinje cells exhibited behaviours similar to those encountered in ASD patients, including abnormal social interaction, repetitive behaviour, and vocalisations. Furthermore, they were reported to have a decreased Purkinje cell excitability. Interestingly, treatment with rapamycin (a mTOR inhibitor) prevented mice from showing any ASD-like behaviours. These findings demonstrate that there is a direct link between cerebellar disruption and cognitive disorders such as ASD.

Reith et al. (2013) performed a similar study to the one presented above. Despite using mice with an induced TSC disorder, their new TSC mutant mouse model was designed to display a specific Purkinje cell TSC2 loss. In the TSC2 mutant mice Purkinje cell degeneration developed during the course of the study. After the induced impairment of Purkinje cells, Reith et al. (2013) conducted a series of behavioural tests to see if TSC2 mice displayed any ASD symptomatology. TSC2 mutant mice showed increased ASD-like behaviours, such as repetitive burying of objects. Similarly to Tsai et al.'s (2012) results, Reith et al. (2013) reported that TSC2 mice showed behavioural and social deficits, such as having no preference between a member of the same species and a random object, or between familiar and unfamiliar mice. These results show that Purkinje cell abnormalities are sufficient to exhibit behaviours similar to those in ASD. It is important to note that, when treated with rapamycin, mice did not display any ASD-like behaviours. Reith et al.'s (2013) study showed that autistic-like behavioural deficits might be induced through the loss of TSC2 in Purkinje cells.

In summary, Reith et al. (2013) demonstrated that TSC2 in Purkinje cells is responsible for deficits in social behaviour and increased repetitive behaviours. Their study is crucial in Purkinje cell and autism research because it established a new TSC mouse model that has the potential to study cerebellar disruption and allows the study of autistic-like behaviours in mice. Similar to Tsai et al.'s (2012) results, this research provides evidence that disruption in Purkinje cells may be an important link between tuberous sclerosis and ASD.

Studies have also revealed mutations on chromosome 10 (PTEN) in individuals with ASD (Butler et al. 2005; Cupolillo et al. 2016). More specifically, the phosphatase and tensin homolog (PTEN) was missing. Cupolillo et al. (2016) in their study analysed the link between cerebellar Purkinje cell PTEN loss and autism. Using a targeted DNA modification system, they created a special mutant mouse in which phosphatase and tensin homolog inactivation was generated for cerebellar Purkinje cells. Cupolillo et al.'s (2016) adult Purkinje cell PTEN-mutant mice have been reported to display both physical and mental deficits. Mutant mice have been reported to be less sociable, display repetitive behaviour, have problems in motor learning, and have less cognitive flexibility (Cupolillo et al. 2016). Such results are in concordance with symptoms reported in Reith et al.'s (2013) and Tsai et al. (2012) mutant mice. Mutant Purkinje cells appeared abnormal. It has also been reported that PTEN plays an important role in regulating Purkinje cell morphology. Cupolillo et al. (2016) stated in their study that the absence of the phosphatase and tensin homolog in a Purkinje cell leads to severe structural abnormalities such as overgrowth of all Purkinje cell compartments, accumulation of cytoskeletal components, both dendritic and axonal swelling, dendritic branching pathologies, and progressive Purkinje cell loss. Cupolillo et al.'s (2016) research gives a better understanding of the role of PTEN in Purkinje cells. This study is the first one to show a connection between Purkinje cell degeneration and loss of PTEN, and autistic spectrum disorder. Those findings add to earlier studies by Tsai et al. (2012) and Reith et al. (2013).

Despite being performed on mice, these animal model studies might make an important contribution to the future treatment of ASD. 
The importance of those studies lies not only in demonstrating the link between the causes of ASD and neurodegeneration, but also it sheds light into new methods of treatment of ASD. For instance, rapamycin used in Reith et al.'s (2013) study is an immunosuppressant widely researched for treating disorders such as TSC and ASD. Despite its known side effects, rapamycin treatment trials are seeking to develop a new drug for both disorders.

\section{In vivo studies: neuroimaging and parental reports}

Contrary to autopsy studies, neuroimaging allows a greater number of individuals to be tested. Unfortunately, in vivo studies involving brain scanning methods are not very well represented in the topic of Purkinje cell loss. Despite the small number of imaging studies, they confirm the results obtained in animal model and autopsy studies.

One of the earliest studies involving neuroimaging of autistic patients is presented in Courchesne et al.'s (1993) research. Their study used MR images of a sample of 21 healthy autistic patients and MR scans of control subjects without any neuroanatomic abnormalities. The age of all tested individuals varied between 5 and 35 years of age. The study reported reduced volume loss in in several brain areas (parietal, superior frontal and occipital lobes, and the corpus callosum). Despite the fact that this early study did not involve the cerebellum, it hypothesised that similar findings might be obtained in that brain area. Furthermore, Courchesne et al.'s (1993) study linked the above-listed cerebral abnormalities with Purkinje neuron loss that, according to them, would have a very early onset (probably in the gestational period), but the first symptoms might be detected only later in life. Furthermore, Courchesne et al. (1993) hypothesised that both genetic and non-genetic causes underlying Purkinje neuron pathologies disrupt neuronal growth in other brain areas.

A more recent study presented by Jeong $e t$ al. (2014) focused on the connection between Purkinje cell loss and ASD. However, previous research has been conducted on the same topic by the same team; the most recent study (Jeong et al. 2014) sums up well the previous findings. In their latest study, Jeong et al. (2014) used a diffusion-weighted MRI tractography on 15 autistic children and 14 healthy controls. The aim of their research was to investigate whether it is possible to detect any structural abnormal- ities in Purkinje cells in living ASD patients. The diffusion-weighted MRI tractography was calibrated to detect any abnormalities in those cerebellar areas that previous post-mortem studies have identified as most at risk of being affected by Purkinje cells loss. Using this method, Jeong et al. (2014) found a reduced number and volume of Purkinje cells across the cerebellum in children with ASD diagnoses compared to their healthy peers. Furthermore, Jeong et al. (2014) findings are very similar to those described in post-mortem studies. This method, however innovative and not without its limitations, confirm that neuroimaging techniques can be used in substitution for older, post-mortem brain studies. There is hope that these new techniques might shed new light on Purkinje cell research.

A small number of studies have sought to investigate the link between neurodegeneration and early onset ASD through parental reports (using questionnaires). For instance, Kern et al. (2002) report that over $80 \%$ of the surveyed parents reported postnatal abnormalities in their children. Those pathologies were supposed to appear before the child received an ASD diagnosis. However, they claimed that most parents noticed early developmental delays in their children, and the results were highly unreliable due to the method used. Despite being non-invasive this research method seemed not to be appropriate.

\section{Limitations and possibilities of future development}

All the above-mentioned studies, regardless of the used method, have shown a significant decrease in the number of Purkinje cells. Reduced numbers of Purkinje cells in the cerebellum have been reported in both children and adults, regardless of their medical condition. Researchers have stated that (Kemper and Bauman 1993; Bailey et al. 1998; Fatemi et al. 2002) Purkinje cell abnormalities in the cerebellar are very likely to have been acquired early in development. These findings, combined with reported age-related changes in brain weight and volume, have raised the possibility that the neuropathology of autism may represent an ongoing process.

The limited number of studies on Purkinje cells and autism might be caused by the expensive methods used and the expertise needed to perform them (mutant animal models, pathological examinations, neuroimaging). Despite the existence of some parental report studies on Purkinje cells and autism (Kern et al. 2002), the efficacy and reliability is questionable. Firstly, 
the developmental abnormalities reported in children after birth are hardly linked with neurodegeneration of a particular brain area (Kern et al. 2002). Secondly, as shown in several other studies based on parental reports, parents might be oversensitive to their child's abnormal development (Glascoe 1994), and the study design might affect their responses (Kroes et al. 2003).

The major weakness of research on Purkinje cells is that the studies that were most successful were performed on mutant rats or brains of deceased individuals. Such techniques are not only expensive, but their results might also vary from those performed on living human brains (due to anatomic and genetic differences). However, despite their limitations, studies on mutant mice have shown positive results. Researchers are thus hopeful that further research involving animals might lead to the development of a new therapy for ASD.

In the academic field there is also hope that new brain imaging techniques will bring new possibilities to research on the neurological basis of autism. Unfortunately, so far only a very limited number of Purkinje cell studies have focused on brain imaging techniques. Undoubtedly, more in vivo studies are needed because new brain scanning techniques seem to be useful in the future in the search for abnormalities in the brain area. Those shown by the Jeong research group (Jeong et al. 2014) have satisfactory results in linking Purkinje cells with ASD, and they involve the brains of living human ASD patients. Further research might be focused on other brain regions as well, to compare previous results. Furthermore, it is a better understanding of the role of each of the cerebellar regions is needed. The aim of future research will undoubtedly be the further study of the relationship between cerebellar impairment and autism spectrum disorder.

\section{Conclusions}

In the human brain there are different types of neurons that have different characteristics, and they respond differently to intoxication or injury. Purkinje cells in the cerebellum have been documented to play an important role; however, they are highly vulnerable to any type of insult. Unfortunately, the mechanisms that produce Purkinje neuron loss in autism remain unknown. What is known is that their abnormal functioning is closely linked with autism spectrum disorder symptomatology. Autism (both adult and infantile) is a behaviourally-defined condition associated with disrupted social interaction, communication, and specific behaviours.

All the studies discussed above (mutant mice models, post-mortem examinations, and in vivo neuroimaging methods) have confirmed the presupposition that Purkinje cells and autism are linked. There is hope in the implementation of brain imaging methods. Unfortunately, due to them being expensive, at this point only a very limited number of studies have tried to implement this technique.

Despite the consistency of findings suggesting that Purkinje cell loss and autism might have a common aetiology, more research is needed to investigate this statement. In addition, several researchers have suggested that the complexity of autism spectrum disorder might suggest that there are several coexisting causes of the disorder.

\section{Acknowledgements}

I would like to thank Dr Ines Jentzsch from the School of Psychology and Neuroscience, St Andrews, and Prof. Tomasz Wolańczyk from the Department of Child Psychiatry, Warsaw Medical University, who provided me with precious feedback and support.

\section{References}

1. American Psychiatric Association. Diagnostic And Statistical Manual Of Mental Disorders, Fifth Edition. DSM-5. New York 2014.

2. Au AK, Chen Y, Du L, et al. Ischemia-induced autophagy contributes to neurodegeneration in cerebellar Purkinje cells in the developing rat brain and in primary cortical neurons in vitro. Biochim Biophys Acta 2015; 1852: 1902-1911.

3. Bailey A, Luthert P, Dean A, et al. A clinicopathological study of autism. Brain 1998; 121: 889-905.

4. Beversdorf DQ, Manning SE, Hillier A, et al. Timing of prenatal stressors and autism. J Autism Dev Disord 2005; 35: 471-478.

5. Butler MG, Dasouki MJ, Zhou XP, et al. Subset of individuals with autism spectrum disorders and extreme macrocephaly associated with germline PTEN tumour suppressor gene mutations. J Med Genet 2005; 42: 318-321.

6. Chrobak AA, Soltys Z. Bergmann glia, long-term depression, and autism spectrum disorder. Mol Neurobiol 2017; 54: 1156-1166.

7. Courchesne E, Press GA, Yeung-Courchesne R. Parietal lobe abnormalities detected with MR in patients with infantile autism. Am J Roentgenol 1993; 160: 387-393.

8. Cupolillo D, Hoxha E, Faralli A, et al. Autistic-like traits and cerebellar dysfunction in Purkinje cell PTEN knock-out mice. Neuropsychopharmacology 2016; 41: 1457.

9. Dapretto M, Davies MS, Pfeifer JH, et al. Understanding emotions in others: mirror neuron dysfunction in children with autism spectrum disorders. Nat Neurosci 2006; 9: 28. 
10. Fatemi SH, Halt AR, Realmuto G, et al. Purkinje cell size is reduced in cerebellum of patients with autism. Cell Mo Neurobiol 2002; 22: 171-175.

11. Finger S. Origins of neuroscience: a history of explorations into brain function. Oxford University Press, New York 2001

12. Glascoe FP. It's not what it seems: The relationship be tween parents' concerns and children with global delays. Clin Pediatr 1994; 33: 292-296.

13. Gross CG. Leonardo da Vinci on the brain and eye. Neuroscientist 1997; 3: 347-355.

14. Hampson DR, Blatt GJ. Autism spectrum disorders and neuropathology of the cerebellum. Front Neurosci 2015; 9: 420.

15. Hardan AY, Muddasani S, Vemulapalli M, et al. An MRI study of increased cortical thickness in autism. Am J Psychiatry 2006; 163: 1290-1292.

16. Jeong JW, Tiwari VN, Behen ME, et al. In vivo detection of reduced Purkinje cell fibers with diffusion MRI tractography in children with autistic spectrum disorders. Front Hum Neurosci 2014; 8: 110

17. Joachim CL, Morris JH, Selkoe DJ. Diffuse senile plaques occur commonly in the cerebellum in Alzheimer's disease. Am J Pathol 1989; 135: 309.

18. Kaur C, Sivakumar V, Zou Z, et al. Microglia-derived proinflammatory cytokines tumor necrosis factor-alpha and interleukin-1beta induce Purkinje neuronal apoptosis via their receptors in hypoxic neonatal rat brain. Brain Struct Funct 2014; 219: 151-170.

19. Kemper TL, Bauman ML. The contribution of neuropathologic studies to the understanding of autism. Neurol Clin 1993; 11: 175-187.

20. Kern JK, Jones AM. Evidence of toxicity, oxidative stress, and neuronal insult in autism. J Toxicol Environ Health 2006; 9: 485-499.

21. Kern JK, Miller VS, Evans PA, et al. Efficacy of porcine secretin in children with autism and pervasive developmental disorder. J Autism Dev Disord 2002; 32: 153-160.

22. Kroes G, Veerman JW, De Bruyn EE. Bias in parental reports? Maternal psychopathology and the reporting of problem behavior in clinic-referred children. Eur J Psychol Assess 2003: 19: 195

23. Krook-Magnuson E, Szabo GG, Armstrong C, et al. Cerebellar directed optogenetic intervention inhibits spontaneous hippocampal seizures in a mouse model of temporal lobe epilepsy. eNeuro 2014; 1: 2-16.

24. Li WK, Hausknecht MJ, Stone P, et al. Using a million cell simulation of the cerebellum: Network scaling and task generality. Neural Netw 2013; 47: 95-102.

25. Ludlow M, Kortekaas J, Herden C, et al. Neurotropic virus infections as the cause of immediate and delayed neuropathology. Acta Neuropathol 2016; 131: 159-184.

26. McPartland JC, Reichow B, Volkmar FR. Sensitivity and specificity of proposed DSM-5 diagnostic criteria for au tism spectrum disorder. J Am Acad Child Adolesc Psychiatry 2012; 51: 368-383.

27. Munson J, Dawson G, Abbott R, et al. Amygdalar volume and behavioral development in autism. Arch Gen Psychiatry 2006; 63: 686-693.

28. Petropoulos H, Friedman SD, Shaw DW, et al. Gray matter abnormalities in autism spectrum disorder revealed by T2 relaxation. Neurology 2006; 67: 632-636.

29. Reith RM, McKenna J, Wu H, et al. Loss of Tsc2 in Purkinje cells is associated with autistic-like behavior in a mouse model of tuberous sclerosis complex. Neurobiol Dis 2013; 51; 93-103.
30. Ritvo ER, Freeman BJ, Scheibel AB, et al. Lower Purkinje cell counts in the cerebella of four autistic subjects: Initial findings of the UCLA-NSAC autopsy research report. Am J Psychiatry 1986; 43: 862-866.

31. Role L. Autism Synaptic and Cellular Mechanisms. Nanosymposium. Society of Neuroscience, 2015.

32. Rubenstein JLR, Merzenich MM. Model of autism: in creased ratio of excitation/inhibition in key neural systems. Genes Brain Behav 2003; 2: 255-267.

33. Schmahmann JD, Sherman JC. The cerebellar cognitive affective syndrome. Brain 1998; 121: 561-579.

34. Shattuck PT. The contribution of diagnostic substitution to the growing administrative prevalence of autism in US special education. Pediatrics 2006; 7: 1028-1037.

35. Sowell ER, Jernigan TL, Mattson SN, et al. Abnormal development of the cerebellar vermis in children prenatally exposed to alcohol: size reduction in lobules I-V. Alcohol Clin Exp Res 1996; 20: 31-34.

36. Starowicz-Filip A, Milczarek O, Kwiatkowski S, et al. Rola móżdżku w regulacji funkcji poznawczych - ujęcie neuropsychologiczne. Neuropsychiatr Neuropsychol 2013; 8: 24-31.

37. Stoodley CJ. The cerebellum and cognition: evidence from functional imaging studies. Cerebellum 2012; 11: 352-365.

38. Sudarov A. Defining the role of cerebellar Purkinje cells in autism spectrum disorders. Cerebellum 2013; 12: 950-955.

39. Takashima S. Olivocerebellar lesions in infants born prematurely. Brain Dev 1982; 4: 361-366.

40. Traut N, Beggiato A, Bourgeron T, et al. Cerebellar volume in autism: Meta-analysis and analysis of the ABIDE COhort. Biol Psychiatry 2018; 83: 579-588.

41. Tsai PT, Hull C, Chu Y, et al. Autistic-like behaviour and cerebellar dysfunction in Purkinje cell Tsc1 mutant mice. Nature 2012; 488: 647

42. Ulatowski L, Parker R, Warrier G, et al. Vitamin E is essential for Purkinje neuron integrity. Neurosci 2014; 260: 120-129.

43. Voogd J, Glickstein M. The anatomy of the cerebellum. Trends Cogn Sci 1998; 2: 307-313.

44. Wallace GL, Dankner N, Kenworthy L, et al. Age-related temporal and parietal cortical thinning in autism spec trum disorders. Brain 2010; 133: 3745-3754.

45. Whitney ER, Kemper TL, Rosene DL, et al. Density of cerebellar basket and stellate cells in autism: evidence for a late developmental loss of Purkinje cells. J Neurosci Res 2009; 87: 2245-2254.

46. Yamada K, Watanabe M. Cytodifferentiation of Bergmann glia and its relationship with Purkinje cells. Anat Sci Int 2002; 77: 94-108.

47. Yip J, Soghomonian JJ, Blatt GJ. Decreased GAD67 mRNA levels in cerebellar Purkinje cells in autism: pathophysiological implications. Acta Neuropathol 2007; 113: 559-568. 